\title{
USP26 deubiquitinates androgen receptor (AR) in the maintenance of sperm maturation and spermatogenesis through the androgen receptor signaling pathway
}

\author{
Jing Wang ${ }^{A}$, Xia Zhao ${ }^{B}$, Renyun Hong ${ }^{C}$, Jing Wang ${ }^{D-F}$ \\ Department of Reproductive Medicine, Zhongda Hospital, School of Medicine, Southeast University, Nanjing, China \\ A - research concept and design; B - collection and/or assembly of data; C - data analysis and interpretation; \\ $D$ - writing the article; $E$ - critical revision of the article; $F$ - final approval of the article
}

Address for correspondence

Jing Wang

E-mail: waterquiet1006@126.com

\section{Funding sources}

Southeast University (Nanjing, China) Basic Research Business Fee Basic Support Project: (grants No. 2242019K40266 and 2242019K40244). The National Natural Science Foundation of China (grant No. 81901469) funding.

\section{Conflict of interest}

None declared

\section{Acknowledgements}

This work was supported by the Zhongda Hospital, School of Medicine, Southeast University, Nanjing, China.

Received on February 18, 2020

Reviewed on March 17, 2020

Accepted on June 2, 2020

Published online on October 16, 2020

\begin{abstract}
Background. The post-translational modifications of proteins control various physiological and pathological events in cells.
\end{abstract}

Objectives. In this study, we investigated the influences of the X-linked deubiquitination enzyme USP26 in mediating androgen receptor (AR) deubiquitination in the physiological events of sperm maturation and spermatogenesis through the AR signaling pathway.

Material and methods. The cell cycle results detected with flow cytometry (FCM) showed that both of the proteins, USP26 and AR, could facilitate the transition of G1-G2 phase in the Leydig cells (TM3). This effect also promoted the proliferation of the Leydig cells.

Results. The cell cycle studies performed using FCM detected that the 2 proteins, USP26 and AR, could facilitate the transition of $\mathrm{G} 1-\mathrm{G} 2$ phase in the Leydig cells (TM3). This effect also promoted the proliferation of the Leydig cells. Moreover, the results from co-immunoprecipitation (CO-IP), immunofluorescence and western blot assays showed that the physiological process due to USP26 interacted with AR and influenced AR deubiquitination, thus upregulating the proteins CCND1 and SPATA46 - which are associated with cell cycle progression and spermatogenesis - as well as decreasing the expression of TP73. Thus, these processes took place through the AR signaling pathway. Furthermore, the USP26 mimic plasmid transfection enhanced these activities, while, conversely, USP26 and AR inhibitor plasmid transfection suppressed the physiological events.

Conclusions. Taken together, the effects of AR deubiquitinated by USP26 could modulate sperm maturation and spermatogenesis through the androgen receptor signaling pathway.

Key words: deubiquitination, sperm maturation, USP26
Cite as

Wang J, Zhao X, Hong R, Wang J. USP26 deubiquitinates androgen receptor (AR) in the maintenance of sperm maturation and spermatogenesis through the androgen receptor signaling pathway. Adv Clin Exp Med. 2020;29(10):1153-1160. doi:10.17219/acem/123355

DOI

10.17219/acem/123355

\section{Copyright}

Copyright by Author(s)

This is an article distributed under the terms of the Creative Commons Attribution 3.0 Unported (CC BY 3.0) (https://creativecommons.org/licenses/by/3.0/) 


\section{Introduction}

The ubiquitination and deubiquitination of protein post-translational modifications play important roles in numerous physiological processes, such as DNA damage sensing and repair, signal transduction, cell proliferation, protein functions, etc. ${ }^{1,2}$ The process of sperm maturation and spermatogenesis requires successive processes of cell mitosis, meiosis and post-meiosis, cell proliferation, gene transcription, gene translation, and protein modification, among others. ${ }^{3}$ Thus, the ubiquitin system is identified as being engaged in the process of spermatid metamorphosis and is present in the human sperm centrosome. ${ }^{4}$

The ubiquitin system contains 3 kinds of enzymes: ubiquitin-activating enzyme (E1), ubiquitin carrier proteins (E2) and ubiquitin protein ligases (E3); additionally, there are 2 structural types of the ubiquitin system: polyubiquitination and monoubiquitination. ${ }^{5}$ The deubiquitinating enzymes consist of the ubiquitin C-terminal hydrolases $(\mathrm{UCH})$ and the ubiquitin-specific processing proteases. ${ }^{6}$ The deubiquitinated enzymes keep the ubiquitin system in balance in physiological events. ${ }^{7}$

The polyubiquitin enzyme Ubi-p63E ${ }^{8}$ and the enzyme USP9X ${ }^{9}$ were proved to be involved in male meiotic cell cycle progression and germ cell differentiation. While the ubiquitin-specific protease 26 (USP26) belongs to the family of deubiquitinating enzymes, it also exhibits deubiquitinating activity when the ubiquitin carboxy-terminal hydrolase $(\mathrm{UCH})$ motif is present and the protein sequence is homology. ${ }^{10}$ Komander et al. ${ }^{11}$ showed that USP26 was responsible for maintaining ubiquitin homeostasis of the cells; it has also been found to be highly expressed in type A and type B spermatogonia, spermatocytes and Leydig cells, etc. ${ }^{10,12}$ Moreover, a study by Christensen et al. ${ }^{13}$ and Ribarski et al. ${ }^{14}$ demonstrated that the ubiquitin enzyme USP26 was essential in the biological activity of spermatogenesis, such as sperm cell mitotic division and maturation, etc.

The process of spermatogenesis begins in puberty and the main androgenic hormone, testosterone, is crucial for the initiation and maintenance of spermatogenesis, ${ }^{15}$ which is mainly through binding to the androgen receptor (AR). Studies have also shown that AR was essential for spermatogenesis and testis development, which is highly expressed in the Sertoli cells and peritubular myoid cells within the testicular tubules. ${ }^{16,17}$ Importantly, the AR signaling pathway is mainly responsible for germ cell mitosis and meiosis, and the maintenance of spermatogenesis. ${ }^{17}$ Dirac et al. ${ }^{18}$ reported that USP26 was a regulator of AR and that it reversed the ubiquitination of AR in the AR signaling pathway. Therefore, in this study, we intended to study the mechanism of sperm maturation and spermatogenesis in the USP26-mediated AR signaling pathway.

\section{Material and methods}

\section{In vitro cell culture and plasmid transfection}

Leydig cells (TM3) and 293T cell line cells were plated in a cell culture flask and cultured in a cell incubator in Dulbecco's modified Eagle's medium (DMEM; Hyclone; Logan, USA) with 5\% fetal bovine serum (FBS; Gibco, Waltham, USA), $100 \mathrm{U} / \mathrm{mL}$ of penicillin, and $100 \mu \mathrm{g} / \mathrm{mL}$ of streptomycin (Sigma-Aldrich, St. Louis, USA) with 5\% $\mathrm{CO}_{2}$ at $37^{\circ} \mathrm{C}$. In addition, an overexpressed USP26 plasmid and a small interfering RNA plasmid constructed by Gemma Bio-technology Co. Ltd. (Shanghai, China) were used. The methods of plasmid transfection were as follows: firstly, the Lipofectamine 2000 and the constructed DNA plasmid with DMEM were separately dissolved without FBS at a ratio of 1:0.05; then, they were mixed together. Once the cells had grown, they were washed twice in phosphatebuffered saline (PBS). Then, the constructed ov-USP26 plasmid, si-USP26, si-LacZ, si-AR, and si-con plasmids were separately transfected into the cells for $12 \mathrm{~h}$.

\section{Flow cytometry analysis}

Leydig cells (TM3) were cultured and the ov-USP26 plasmid, si-USP26, si-AR, and si-con plasmids were transfected as above, and 1 group of TM3 cells was used as a normal control. Then, the cells were collected into tubes once the cell density had reached over $60 \%$, and they were washed in PBS 3 times; $5 \mathrm{~mL}$ of cold ethyl alcohol (70-80\% concentration) was added for $4 \mathrm{~h}$ to stain the cells; later, the cells were washed in PBS twice. Afterwards, the cells were resuspended with $0.5 \mathrm{~mL}$ of mixed PI/RNase staining solution (Beyotime, Beijing, China; c1052), and they were incubated at $4^{\circ} \mathrm{C}$ for $2 \mathrm{~h}$; the cell cycles in G0/G1, S and G2/M phases were analyzed with a Caliber FACS system (Becton Dickinson Biosciences, Franklin Lakes, USA).

\section{Cell proliferation with EdU assay}

Leydig cells (TM3) were plated in a 96-well plate $(5,000 /$ well). Then, the constructed ov-USP26, si-USP26, si-AR, and si-con plasmids were separately transfected into the cell models for $12 \mathrm{~h}$, while 1 group of TM3 cells was used as a normal control. Later, $100 \mu \mathrm{L}$ of EdU (Invitrogen, Carlsbad, USA) was added to the cells and they were incubated for $2 \mathrm{~h}$ at $37^{\circ} \mathrm{C}$; then, they were washed in PBS and fixed in $4 \%$ formaldehyde for $30 \mathrm{~min}$, after which they were removed from the medium. At this point, $50 \mu \mathrm{L}$ of glycine was added to each well in the plate, and the cell plates were shaken in a table concentrator for $5 \mathrm{~min}$. The plates were then removed from the medium and washed in PBS and 0.5\% Triton X-100 at room temperature for $20 \mathrm{~min}$. Then, the cells were washed with BSA buffer and incubated with DAPI solution (1:1000; Sigma-Aldrich) at room temperature in the dark for $30 \mathrm{~min}$. 
After incubation, the medium was removed and the cells were washed in PBS; the cell graphs were then observed with a fluorescent microscope (Leica, Wetzlar, Germany). Additionally, the ratio of EdU-positive cells (red) to total DAPIpositive cells (blue) was analyzed with Image-Pro Plus (Media Cybernetics, Rockville, USA).

\section{Co-immunoprecipitation}

Leydig cells (TM3) were cultured as above and the constructed ov-USP26, si-USP26 and si-con plasmids were separately transfected into the cells for $12 \mathrm{~h}$, until the cell density reached over $70 \%$. Then, the total proteins were collected with RIPA buffer containing phenylmethylsulfonyl fluoride (PMSF). Protein A/G sepharose beads were pre-incubated with anti-USP26 or anti-AR antibody for 1-1.5 $\mathrm{h}$ with balanced wheel rotation. Then, the beads were suspended in the protein lysate medium with balanced wheel rotation overnight at $4^{\circ} \mathrm{C}$. Afterwards, the beads were washed 3 times and centrifuged at 3,000 g to obtain the immunoprecipitates, which were then analyzed using the western blot method.

\section{Immunofluorescence}

Leydig cells (TM3) were plated in a 96-well plate $(5,000 /$ well). Then, the constructed ov-USP26, si-USP26 and sicon plasmids were separately transfected into the cell models for $12 \mathrm{~h}$, while 1 group of TM3 cells was used as a normal control. Then, a cell slide was made for each group and they were fixed with $4 \%$ polyformaldehyde for $30 \mathrm{~min}$. Afterwards, the cells were permeabilized with $0.1 \%$ TritonX-100 for 5 min and blocked with $10 \%$ bovine serum albumin (BSA) for $60 \mathrm{~min}$ at $37^{\circ} \mathrm{C}$. Finally, the cells were incubated with USP26 (Abcam, Cambridge, UK; 101650) and SPATA46 (Thermo Fisher Scientific, Waltham, USA; $\mathrm{OTI}_{2} \mathrm{~A}_{9}$ ) antibodies for $12 \mathrm{~h}$ at $4^{\circ} \mathrm{C}$. Then, the cells were washed in PBS and incubated with Alexa Fluor 488- (green) and 594- (red) conjugated anti-mouse IgG (Invitrogen) at room temperature for $1 \mathrm{~h}$. The cell nuclei were dyed with DAPI (blue), and the cell slide was mounted with an antifade mounting medium. Then, the images were visualized with a confocal microscope (Leica).

\section{Western blot}

Leydig cells (TM3) and 293T cell line cells were cultured as above; the $293 \mathrm{~T}$ cell line was a control cell line and the constructed ov-USP26, si-USP26, si-LacZ, si-AR, and si-con plasmids were separately transfected into cells for $12 \mathrm{~h}$ until the cell density reached over $70 \%$. One group of TM3 cells was used as a normal control; the LacZ gene was steadily expressed in the cell lines. Then, the total proteins in each group were collected with RIPA buffer containing PMSF protease inhibitors (Beyotime) and the protein concentration was measured. Afterwards, the proteins were denatured with hot water and separated with SDS polyacrylamide gels. Next, the gels were transferred to polyvinylidene difluoride (PVDF) membranes and blocked with $5 \%$ fat-free milk; the membranes were incubated with primary antibodies USP26 (Abcam), AR (Abcam), SPATA46 (Thermo Fisher Scientific), CCND1 (Abcam), TP73 (Abcam), and $\beta$-actin for $12 \mathrm{~h}$; then, the membranes were immunoblotted with anti-mouse IgG antibody for $1 \mathrm{~h}$; finally, the bands were visualized with the chemiluminescent ECL substrate in bandscan and the gray intensity of the proteins was analyzed with ImageJ Software (National Institutes of Health, Bethesda, USA).

\section{Statistical analysis}

The data were analyzed using one-way analysis of variance (ANOVA) in SPSS v. 19.0 statistical software (IBM Corp. Armonk, USA), with a p-value $<0.05$ representing significant differences. Then, the differences between groups were graphed with GraphPad Prism v. 6.0 software (GraphPad Software Inc., San Diego, USA).

\section{Results}

\section{AR deubiquitinated with USP26 could facilitate Leydig cell division and proliferation}

To determine the functional role of USP26 in Leydig cells, we individually transfected the ov-USP26, si-USP26 and si-con plasmids into the TM3 cell groups. The cell cycles and cell proliferation characteristics in each group were then assessed, with the results showing that USP26 could promote the transition of G1 phase to G2 (Fig. 1A), that the cell numbers were also increased with ov-USP26 plasmid transfection (Fig. 2A) and that these processes were inhibited with the si-USP26 and si-AR plasmids (Fig. 1A,2A). Additionally, the differences in cell cycle between the si-USP26-transfected group and the si-con and normal control groups were statistically significant; besides, the differences between the ov-USP26-transfected group and other groups were statistically significant $(\mathrm{p}<0.05$; Fig. $1 \mathrm{~B})$, while the differences in cell proliferation between the ov-USP26-transfected group and the si-USP26 and normal control groups were also significant $(\mathrm{p}<0.05$; Fig. 2B). Furthermore, we also transfected the TM3 cells with the constructed si-AR plasmid, and the results suggested that AR mediated the cell cycle transition and cell proliferation, and that the cell cycles and cell proliferation features were inhibited by si-AR plasmid transfection (Fig. 1C,2C). There were significant differences between the si-AR group and the si-con and normal control groups ( $<<0.05$; Fig. 1D,2D).

Moreover, the co-immunoprecipitation (CO-IP) found that USP26 co-mediated AR in the process (Fig. 3A,B). 
A
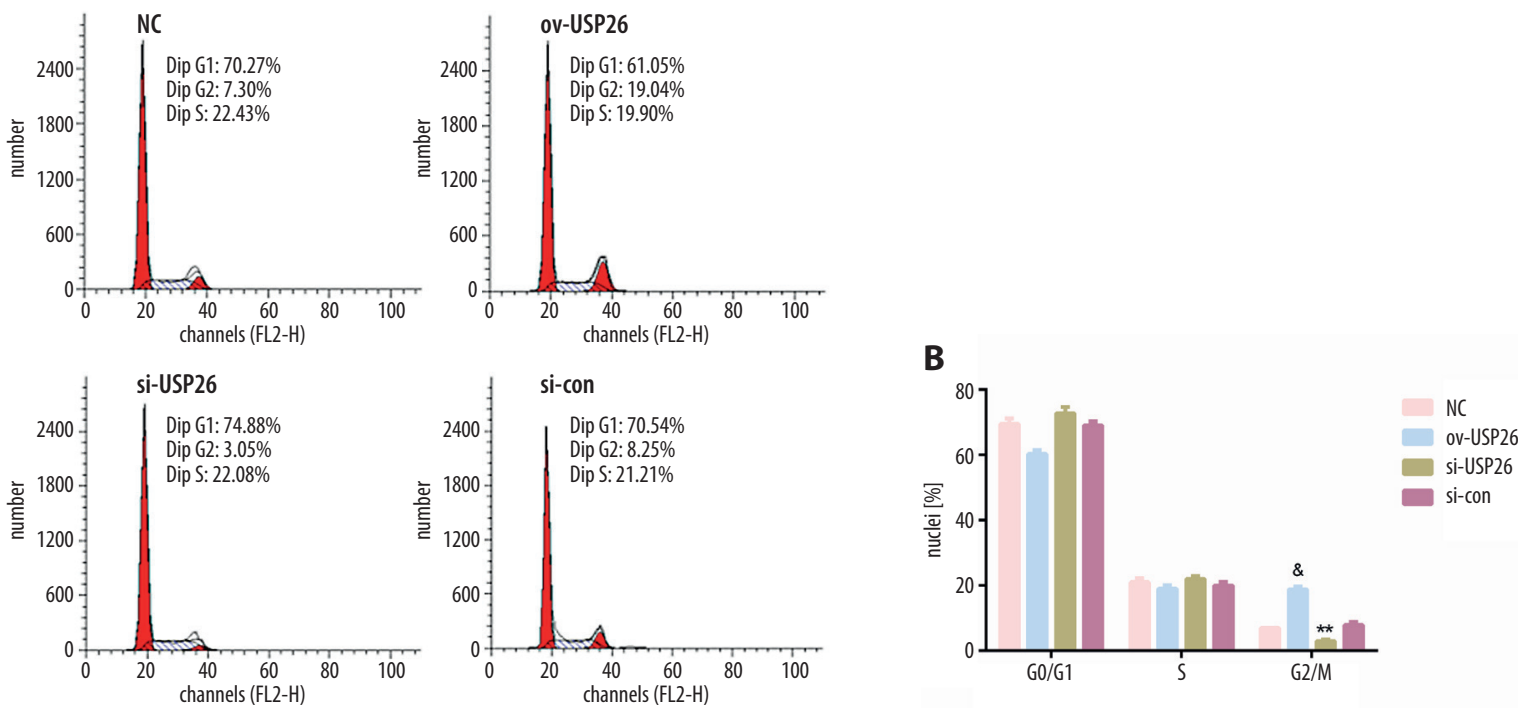

C
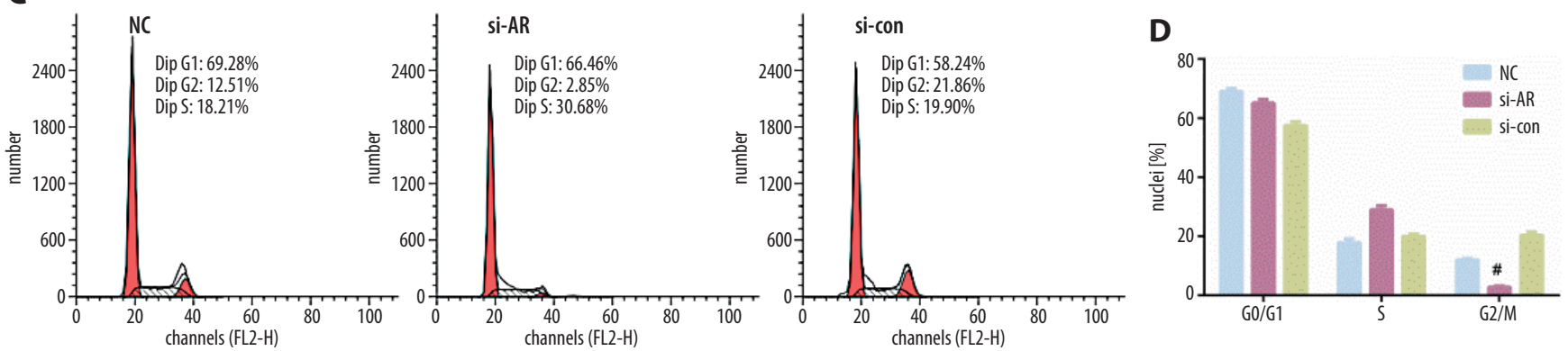

Fig. 1. The proteins USP26 and AR facilitate the cell cycle transition of phase G2 to M. A. Separately transfected into TM3 cells, the ov-USP26, si-USP26 and si-con plasmids varied the cell cycle by group. B. During the G2/M phase, the difference of DNA content in the ov-USP26 plasmid group was higher than in the other groups \& $(p<0.05)$, and in the ov-USP26 group compared with the other groups $(p<0.001)$, while the DNA content was the lowest in the siUSP26 plasmid group when compared with the NC and si-con plasmid groups ${ }^{* *}(p<0.05)$. C. The cell cycle transition of the G2/M phase was inhibited with si-AR plasmid transfection. D. The difference of DNA content in the si-AR plasmid group was lower than in the other 2 groups $\#(p<0.05)$. Mean values and standard deviation (SD) are presented as vertical bars $(n=3)$

Therefore, the deubiquitinating USP26 deubiquitinated AR directly in the process of Leydig cell division and proliferation.

\section{USP26 co-regulated with AR to modulate sperm maturation and spermatogenesis through the androgen receptor signaling pathway}

Immunofluorescence with confocal microscopy was used to detect the proteins USP26 and SPATA46 with a greenor red-conjugated secondary antibody; both of the proteins were visualized directly. Additionally, both of the proteins were located in the nuclear foci, and the merged image showed that the 2 proteins were co-localized (Fig. 4A). Also, the results showed that si-USP26 plasmid transfection could inhibit the expression of the protein SPATA46. A significant difference was found in the expression of the 2 proteins in the ov-USP26-transfected group compared with the si-USP26, si-con transfected groups and the normal control group (\#, \& p < 0.05, Fig. 4B). Moreover, the proteins associated with the AR signaling pathway, such as USP26, AR, SPATA46, CCND1, and TP73 were detected. The results showed that the constructed plasmid was transfected successfully into the TM3 cells and the 293T cell line cells, and that it significantly regulated the expression of the proteins (Fig. 5A-D). Additionally, the proteins USP26, AR, SPATA46, and CCND1 were noticeably upregulated with ov-USP26 plasmid transfection; conversely, si-USP26 plasmid transfection inhibited this effect while upregulating the expression of TP73 that is associated with cell cycle arrest (Fig. 5A,B). In addition, si-AR plasmid transfection also suppressed the expression of the proteins CCND1 and SPATA46, while upregulating the expression of the protein TP73 (Fig. 5C,D). The difference in the proteins among the groups are presented in Fig. 5E-H. The proteins AR, CCND1, and TP73 are part of the AR signaling pathway, hence USP26 deubiquitinated AR to promote sperm maturation and spermatogenesis through the AR signaling pathway. 
A
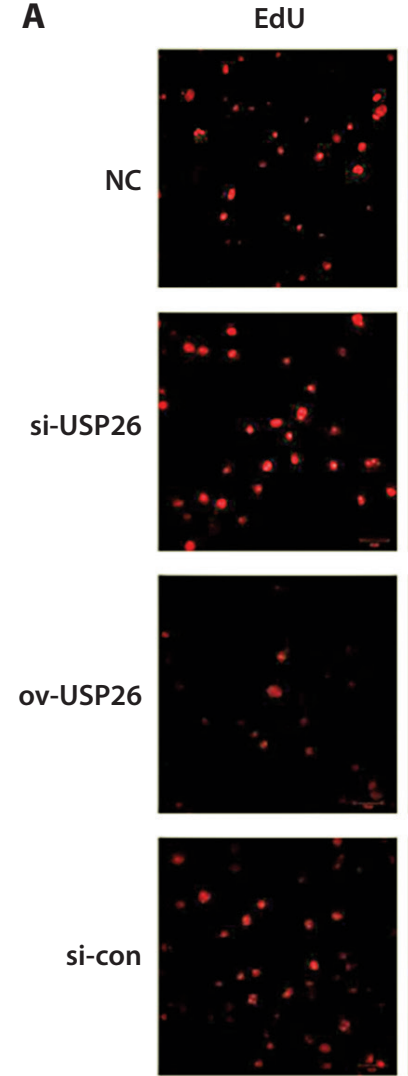

C
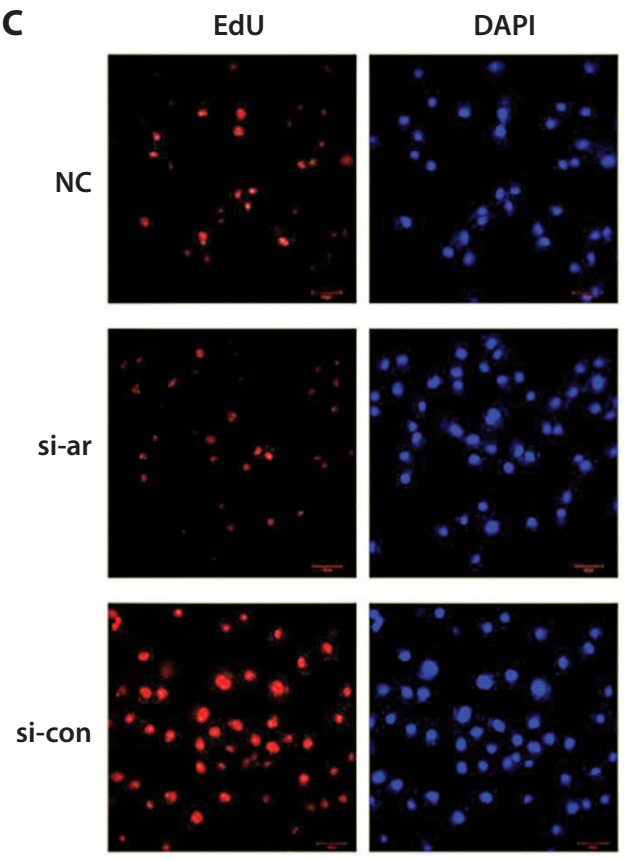

DAPI
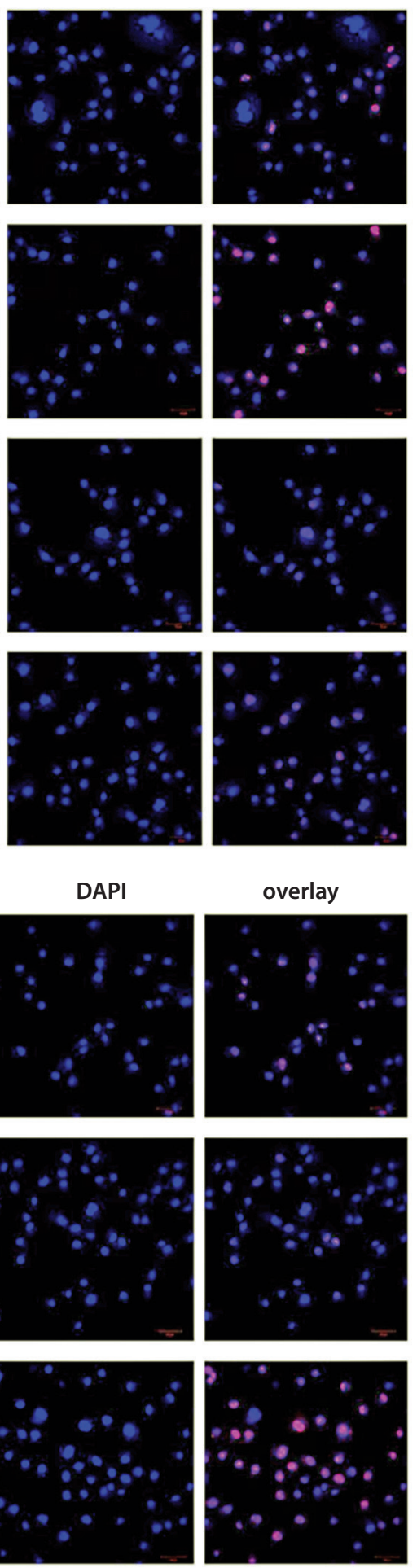

B

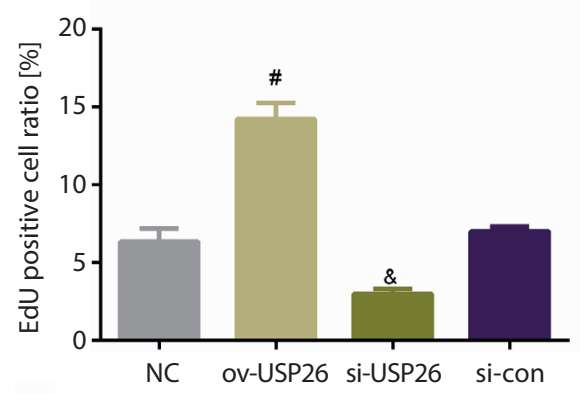

NC

ov-USP26

si-USP26

si-con

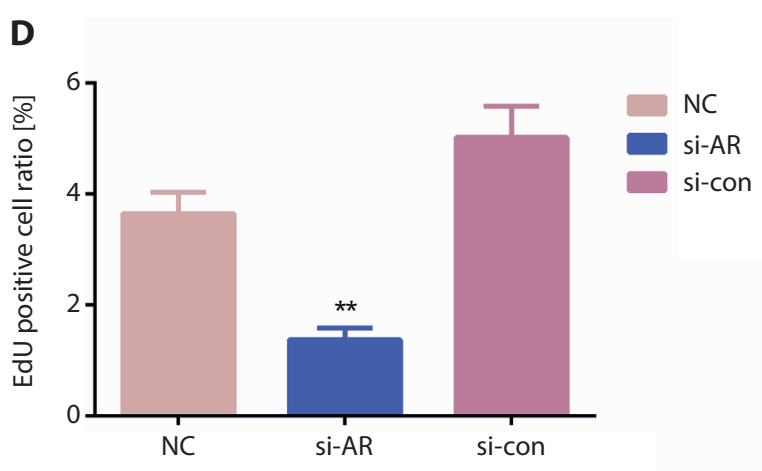

Fig. 2. A and C. EdU proliferation assays of TM3 cells received the ovUSP26, si-USP26, si-AR, and si-con plasmid transfections. B. The USP26 gene promoted cell proliferation $*(p<0.05)$ in the ov-USP26

plasmid group more than in the other groups ${ }^{*}(p<0.05)$ in si-USP26 plasmid group compared with the NC group and si-con plasmid group. D. The cell proliferation rate was inhibited by si-AR plasmid transfection ** $(p<0.05)$ in the si-AR plasmid group more than in the other groups. The mean values and standard deviation (SD) are presented as vertical bars $(n=3)$

A
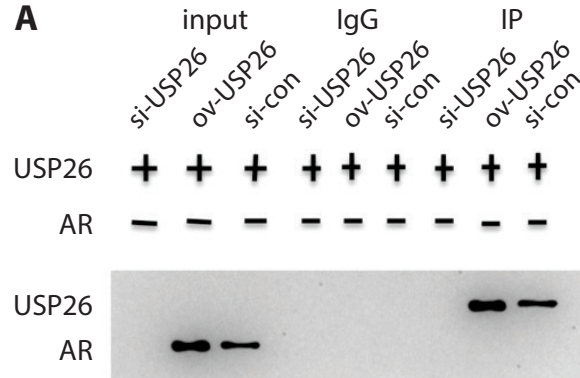

B

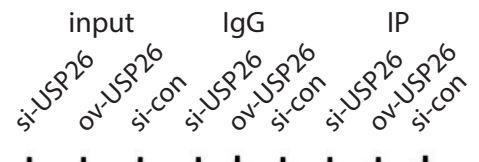

USP $26+\boldsymbol{t}+\boldsymbol{t}+\boldsymbol{t}+\boldsymbol{t}+$

$\mathrm{AR}---\cdots-$

USP26
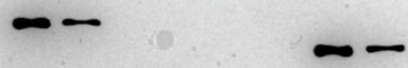

AR
Fig. 3. A and B. The co-immunoprecipitation (CO-IP) was performed in TM3 cells of the groups transfected with ov-USP26, si-USP26 and si-con plasmids. The protein USP26 interacted with AR 
A
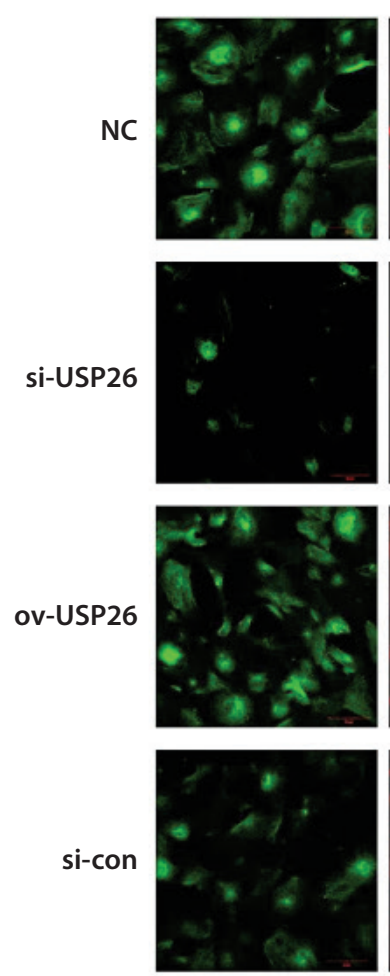

SPATA46
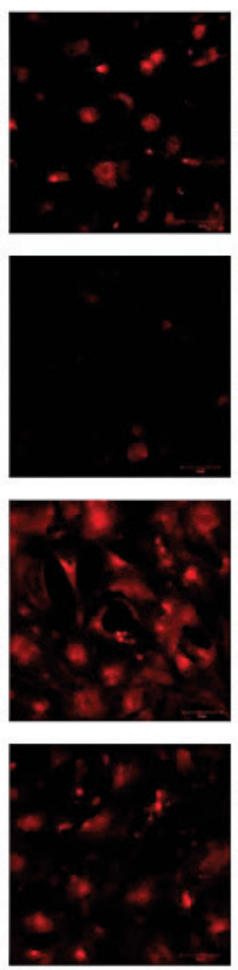

DAPI
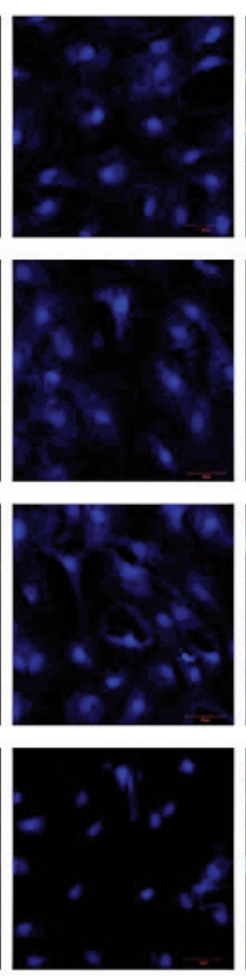

merge
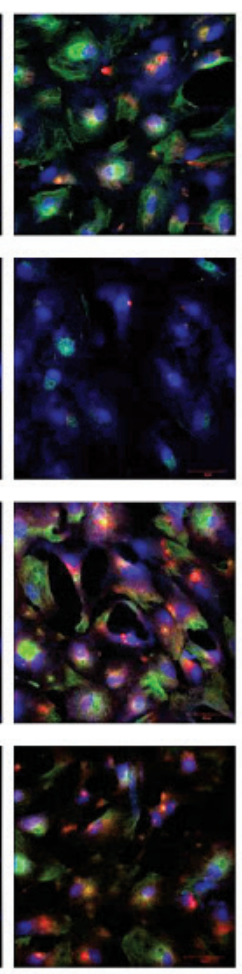

B

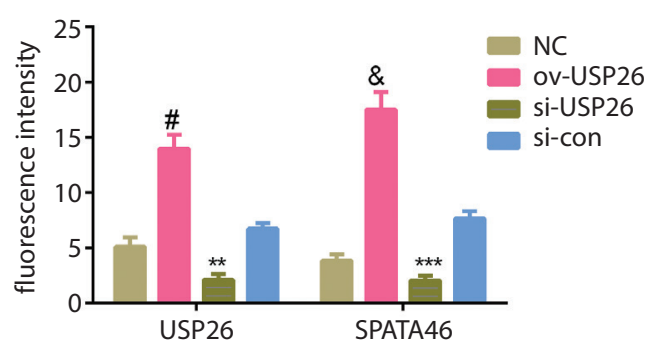

Fig. 4. A. Immunofluorescence staining in TM3 cells, the co-localization of USP26 (green) and SPATA46 (red) was detected in the groups transfected with plasmids. Neither USP26 (green) or SPATA46 (red) was detected in the si-USP26-transfected group, while both the USP26 (green) and SPATA46 (red) proteins were expressed in the TM3 cells transfected with ov-USP26 plasmid. The nuclei were stained with DAPI (blue). Scale bar, $50 \mu \mathrm{m}$. B. Percentage of cells showing USP26 and SPATA46 expression in the different groups $\#, \&(p<0.05)$ in the ov-USP26 plasmid group compared with the other groups ${ }^{* * * * * *}(p<0.05)$ in si-USP26 plasmid group compared with the NC group and si-con plasmid group

\section{Discussion}

Sperm maturation and spermatogenesis is a physiological process that is dependent on the regulation and modification at transcriptional and post-transcriptional levels. ${ }^{19}$ The ubiquitin system has been suggested as being involved in the different phases of spermatogenesis ${ }^{4,20}$; in this study, we found that the deubiquitinating enzyme USP26 played important roles in the process of sperm maturation and spermatogenesis by facilitating the cell cycle transition of phase G2 to $\mathrm{M}$ and cell proliferation in the AR signaling pathway. The USP26 gene is a novel ubiquitin-specific protease gene, located on the $\mathrm{X}$ chromosome and consisting of 835 amino acids, which is a controversial gene in mediating the spermatogenesis and fertility. A study by Felipe-Medina et al. ${ }^{21}$ and Zhang et al. ${ }^{22}$ clearly demonstrated that USP26 was not essential for spermatogenesis and fertility, though many studies reported that USP26 was essential for testis development and spermatogenesis..$^{10,15,23}$ Thus, the deubiquitinating enzyme USP26 is thought to be an important regulator in germ cell development. Importantly, we observed that USP26 monoubiquitinated the AR expressed in Leydig cells in biological events. This finding was consistent with the research Bernards et al. ${ }^{18}$ and Dirac et al. ${ }^{19} \mathrm{~A}$ study by Lahav-Baratz et al. ${ }^{25}$ found that USP26 binds to Mdm 2 through its C-terminal domain to regulate AR in testis development. Therefore, there is a possibility that other E3-ligases were collaboratively engaged in our study.

Although androgens are necessary for male sex development in male secondary sexual characteristics starting from puberty and the AR gene is a key regulator in the process ${ }^{26}$ their mechanism is still not clear. In our study, we found that the proteins USP26, AR, CCND1, TP73, and SPATA46 were expressed in the Leydig cells, and that the siRNA-mediated USP26 and AR knockdown in cultured TM3 cells confirmed the efficiency of the proteins USP26 and AR in promoting sperm maturation and spermatogenesis. Besides, this process was enhanced with overexpression of protein USP26. Moreover, the proteins CCND1 and TP73 were closely associated with the cell cycle transition. ${ }^{27,28}$ In our study, the upregulation of CCND1 and the downregulation of TP73 participated in the process of G2/M phase transition and cell proliferation. To our knowledge, the AR signaling pathway in germ cell development mainly controls the cyclin-dependent kinases (Cdks) at the appropriate time during the cell cycles. ${ }^{17,18,29}$ The AR has also been identified in the regulation of sex determination and differentiation in zebrafish. ${ }^{30}$ Moreover, the protein SPATA46 located on the nuclear membrane was recognized as an important regulator in spermatogenesis in mice. ${ }^{31}$ Therefore, the CCND1, TP73 and SPATA46 genes are possible downstream signals in the AR signaling pathway. However, the direct upstream regulator of SPATA46 still requires further study as part of the AR signaling pathway.

Taken together, USP26 significantly modulated sperm maturation and spermatogenesis by deubiquitinating AR in the AR signaling pathway. 

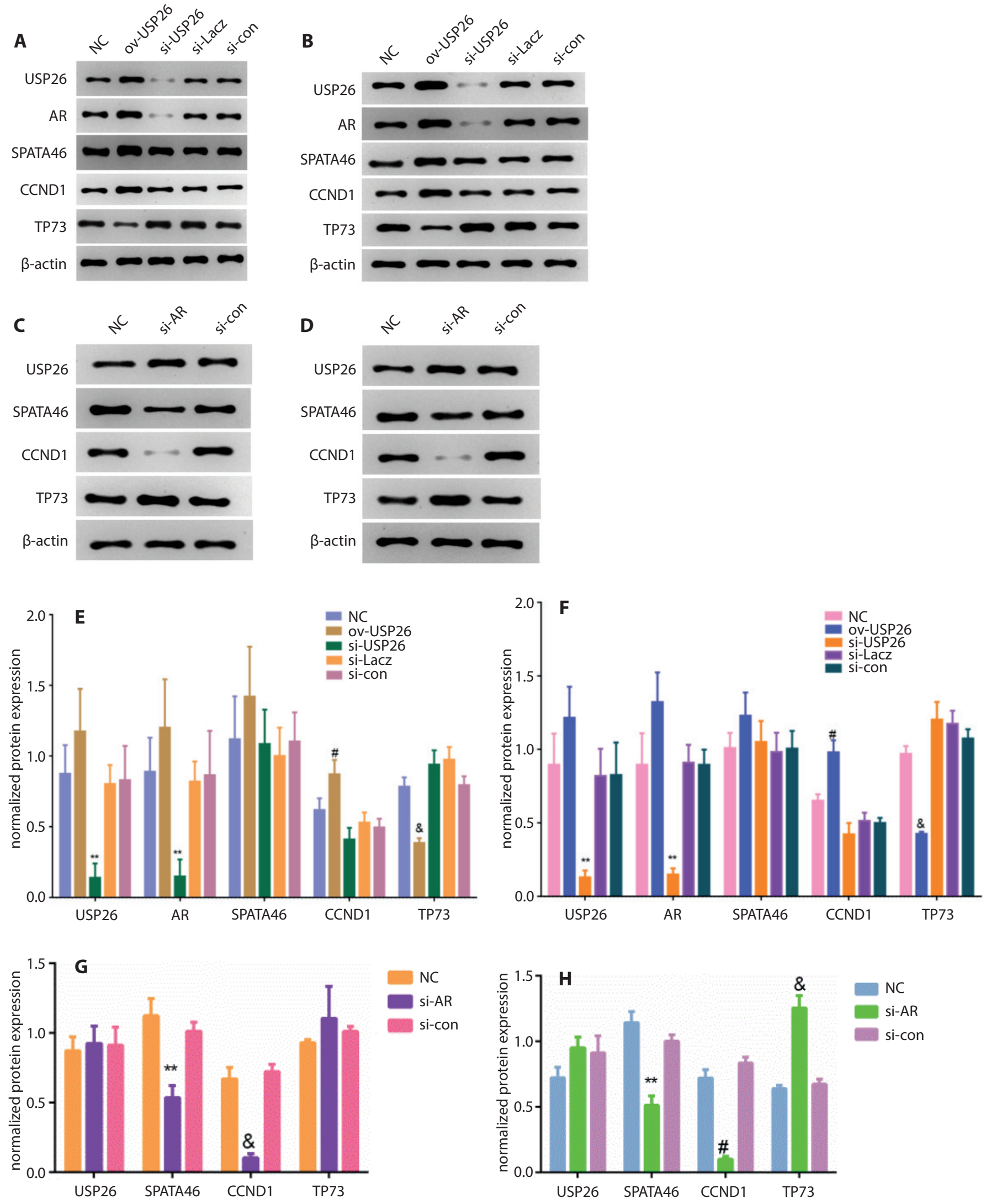

Fig. 5. The USP26, AR, SPATA46, CCND1, and TP73 proteins were detected using the western blot method. A and B. The protein bands represented the protein expression in the TM3 and 293T cell groups transfected with ov-USP26, si-USP26, si-LacZ, and si-con plasmids, along with the normal control group. C and D. The protein bands represent the protein expression in the TM3 and 293T cell groups transfected with si-AR and si-con plasmids, along with the normal control group. E and F. ** $p<0.05$ of USP26 and AR proteins in the si-USP26 plasmid group compared with the other groups, $\#, \&<<0.05$ of proteins CCND1 and TP73 in the ov-USP26 plasmid group compared with the other groups. G and H. ${ }^{* *} p<0.05$ of protein SPATA46 in the si-AR plasmid group compared with the other groups, $\#, \& p<0.05$ of protein CCND1 and TP73 in the si-AR plasmid group compared with the other groups, ${ }^{\circledR} p<0.05$ of protein TP73 in the si-AR plasmid group compared with the other groups 


\section{Conclusions}

USP26 interacted with AR and monoubiquitinated AR to facilitate sperm maturation and spermatogenesis through the AR signaling pathway.

\section{ORCID iDs}

Jing Wang (1) https://orcid.org/0000-0002-5528-9070

Xia Zhao (D) https://orcid.org/0000-0002-4791-8172

Renyun Hong (1) https://orcid.org/0000-0001-5602-1052

\section{References}

1. Finley D, Ciechanover A, Varshavsky A. Ubiquitin as a central cellular regulator. Cell. 2004;116(2 Suppl):S29-S32.

2. Wilkinson KD. Ubiquitination and deubiquitination: Targeting of proteins for degradation by the proteasome. Semin Cell Dev Biol. 2000; 11(3):141-148.

3. Sharpe RM. Regulation of Spermatogenesis. The Physiology of Reproduction. $2^{\text {nd }}$ ed. New York, NY: Raven Press; 1994:1363-1434.

4. Sutovsky P. Ubiquitin-dependent proteolysis in mammalian spermatogenesis, fertilization, and sperm quality control: Killing three birds with one stone. Microsc Res Tech. 2003;61(1):88-102.

5. Liu J, Nussinov R. The role of allostery in the ubiquitin-proteasome system. Crit Rev Biochem Mol Biol. 2013;48(2):89-97.

6. He M, Zhou Z, Shah AA, et al. The emerging role of deubiquitinating enzymes in genomic integrity, diseases, and therapeutics. Cell Biosci. 2016;6:62.

7. Reyes-Turcu FE, Ventii KH, Wilkinson KD. Regulation and cellular roles of ubiquitin-specific deubiquitinating enzymes. Annu Rev Biochem. 2009;78:363-397.

8. Lu C, Kim J, Fuller MT. The polyubiquitin gene Ubi-p63E is essential for male meiotic cell cycle progression and germ cell differentiation in Drosophila. Development. 2013;140(17):3522-3531.

9. Kishi K, Uchida A, Takase HM, et al. Spermatogonial deubiquitinase USP9X is essential for proper spermatogenesis in mice. Reproduction. 2017;154(2):135-143.

10. White-Cooper H, Wosnitzer MS, Mielnik A, Robinson B, Schlegel PN Paduch DA. Ubiquitin-specific protease 26 (USP26) expression analysis in human testicular and extragonadal tissues indicates diverse action of USP26 in cell differentiation and tumorigenesis. PLoS One. 2014;9(6):e98638.

11. Komander D, Clague MJ, Urbe S. Breaking the chains: Structure and function of the deubiquitinases. Nat Rev Mol Cell Biol. 2009;10(8): 550-563.

12. Lin YW, Hsu TH, Yen PH. Localization of ubiquitin specific protease 26 at blood-testis barrier and near Sertoli cell-germ cell interface in mouse testes. Int J Androl. 2011;34(5 Pt 2):e368-e377.

13. Christensen GL, Griffin J, Carrell DT. Sequence analysis of the X-linked USP26 gene in severe male factor infertility patients and fertile controls. Fertil Steril. 2008;90(3):851-852.
14. Ribarski I, Lehavi O, Yogev L, et al. USP26 gene variations in fertile and infertile men. Hum Reprod. 2009;24(2):477-484.

15. McLachlan RI, O'Donnell L, Meachem SJ, et al. Identification of specific sites of hormonal regulation in spermatogenesis in rats, monkeys, and man. Recent Prog Horm Res. 2002;57:149-179.

16. Tan KA, De GK, Atanassova N, et al. The role of androgens in Sertoli cell proliferation and functional maturation: Studies in mice with total or Sertoli cell-selective ablation of the androgen receptor. Endocrinology. 2005;146(6):2674-2726.

17. De Gendt K, Swinnen JV, Saunders PT, et al. A Sertoli cell-selective knockout of the androgen receptor causes spermatogenic arrest in meiosis. Proc Natl Acad Sci U S A. 2004;101(5):1327-1332.

18. Dirac AM, Bernards R. The deubiquitinating enzyme USP26 is a regulator of androgen receptor signaling. Mol Cancer Res. 2010;8(6):844-854.

19. Legrand JMD, Hobbs RM. RNA processing in the male germline: Mechanisms and implications for fertility. Semin Cell Dev Biol. 2018;79: 80-91.

20. Baarends WM, van der Laan R, Grootegoed JA. Specifc aspects of the ubiquitin system in spermatogenesis. J Endocrinol Invest. 2000; 23(9):597-604.

21. Felipe-Medina N, Gomez HL, Condezo YB, et al. Ubiquitin-specific protease 26 (USP26) is not essential for mouse gametogenesis and fertility. Chromosoma. 2019;128(3):237-247.

22. Zhang W, Liu T, Mi YU, et al. Evidence from enzymatic and meta-analyses does not support a direct association between USP26 gene variants and male infertility. Andrology. 2015;3(2):271-279.

23. Tian $\mathrm{H}$, Huo $\mathrm{Y}$, Zhang J, et al. Disruption of ubiquitin specific protease 26 gene causes male subfertility associated with spermatogenesis defects in mice. Biol Reprod. 2019;100(4):1118-1128.

24. Asadpor U, Totonchi M, Sabbaghian M, et al. Ubiquitin-specific protease (USP26) gene alterations associated with male infertility and recurrent pregnancy loss (RPL) in Iranian infertile patients. J Assist Reprod Genet. 2013;30(7):923-931.

25. Lahav-BaratzS, Kravtsova-Ivantsiv Y, Golan S, Ciechanover A. The testisspecific USP26 is a deubiquitinating enzyme of the ubiquitin ligase Mdm2. Biochem Biophys Res Commun. 2017;482(1):106-111.

26. Bukhari I, Li G, Wang L, et al. Effects of androgen receptor mutation on testicular histopathology of patient having complete androgen insensitivity. J Mol Histol. 2017;48(3):159-167.

27. Park S, Park Y, Song G, Lim W. Alpha-solanine inhibits cell proliferation via mitochondrial dysfunction and inhibin synthesis in mouse testis in vitro and in vivo. Chemosphere. 2019;235:271-279.

28. Zamir-Nasta T, Razi M, Shapour H, Malekinejad H. Roles of p21, p53, cyclin D1, CDK-4, estrogen receptor a in aflatoxin B1-induced cytotoxicity in testicular tissue of mice. Environ Toxicol. 2018;33(3-4):385-395

29. Chen SR, Liu YX. Regulation of spermatogonial stem cell self-renewal and spermatocyte meiosis by Sertoli cell signaling. Reproduction. 2015;149(4):R159-R167.

30. Crowder CM, Lassiter CS, Gorelick DA. Nuclear androgen receptor regulates testes organization and oocyte maturation in zebrafish. Endocrinology. 2018;159(2):980-993.

31. Chen J, Gu Y, Zhang Z, et al. Deficiency of SPATA46, a novel nuclear membrane protein, causes subfertility in male mice. Biol Reprod. 2016;95(3):58. 